\title{
A unique uterine cervical "teratocarcinosarcoma": a case report
}

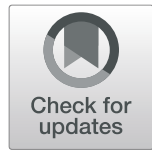

\author{
Kozue Ito ${ }^{1}$, Mitsutake Yano ${ }^{1,2}$, Aiko Ogasawara ${ }^{3}$, Maiko Miwa ${ }^{3}$, Eito Kozawa ${ }^{4}$ and Masanori Yasuda ${ }^{* *}$
}

\begin{abstract}
Background: Teratocarcinosarcoma (TCS) is a rare aggressive tumor of the nasal cavity and paranasal sinuses and has both epithelial and two or more mesenchymal components. In other organs, 5 cases of ovarian tumors closely resembling TCS have been reported; however, there has been no published case of cervical TCS. Herein, we describe a unique case of cervical tumor that had carcinosarcomatous and teratomatous features, resembling a sinonasal TCS.

Case presentation: A 45-year-old woman presented to our hospital for evaluation of a cervical lesion. The gynecologist found a large polypoid mass, whose biopsy showed glandular components of probable germ cell origin based on the immunohistochemical features. The patient underwent total hysterectomy with bilateral salpingo-oophorectomy. The cervical polypoid mass was found to consist of both epithelial and mesenchymal tissues, including immature glandular structure resembling fetal enteric tubules, neuroepithelial cells, hyaline cartilage, and rhabdomyosarcoma cells. This tumor was diagnosed as TCS of the uterine cervix. Following the surgery, the patient received radiotherapy and has been free of disease for 13 months.

Conclusion: This is the first case report of cervical TCS. The tumor is thought to be histogenetically less associated with HPV infection, and its teratomatous components with an absence of cytogenetic abnormalities (including isochromosome 12p (i(12p)) suggest a analogous histogenesis compared to pure mature or immature teratoma.
\end{abstract}

Keywords: Uterine cervix, Teratoma, Carcinosarcoma, Teratocarcinosarcoma

\section{Background}

Carcinosarcoma [1, 2] and teratoma [3, 4] are encountered in the uterine cervix, although rarely. Cervical carcinosarcoma, characterized by a mixture of both malignant epithelial and mesenchymal elements, is an aggressive neoplasm with poor prognosis $[1,2]$. Teratomas consist of two or three germ layers: ectodermal, mesodermal, and endodermal components in any combination. Mature teratoma is usually benign, but $1-3 \%$ cases can show malignant transformation; immature teratoma consists of embryonic tissue, mainly neural tissue. Cervical teratoma is thought to develop from germ cells that get trapped along their migration during embryonic development $[3,5]$.

Teratocarcinosarcoma (TCS) has both epithelial components and two or more mesenchymal components, such as cartilage, fibroblasts, muscular, and bony tissues.

\footnotetext{
*Correspondence: m_yasuda@saitama-med.ac.jp

${ }^{1}$ Department of Pathology, Saitama Medical University International Medical

Center, 1397-1 Yamane, Hidaka-City, Saitama 350-1298, Japan

Full list of author information is available at the end of the article
}

The tumor, known to arise in the nasal cavity and paranasal sinuses, was first described as a teratoid carcinosarcoma by Shanmugaratnam et al. in 1983 [6], whereas the term TCS was initially proposed by Heffner and Hyamsin in 1984 [7]. Since then, there have been less than 100 cases with tumors originating from the nasal cavity or paranasal sinuses reported in the English literature [8]. The patients were predominantly male (87\%) with an average age of 54.5 years $[7,8]$. TCS has several clinicopathological features and problems, such as poor prognosis due to rapid growth, many differential diagnoses, and ambiguities of histogenesis or tumor origin. In sinonasal TCS, radical surgery followed by radiotherapy is the most commonly performed treatment option, however, resulting in a poor prognosis (average survival, 1.7 years) due to frequent recurrence and metastasis [7, 8]. Because TCS shows histologically diverse features such as mature/benign as well as immature / malignant epithelial and mesenchymal components, pathologists need to distinguish TCS from carcinosarcoma, immature

(c) The Author(s). 2019 Open Access This article is distributed under the terms of the Creative Commons Attribution 4.0 International License (http://creativecommons.org/licenses/by/4.0/), which permits unrestricted use, distribution, and 
teratoma, teratoma with malignant transformation, and peripheral primitive neuroectodermal tumor. TCS has been reported to occur in the female reproductive organs, especially in the ovary, other than in the nasal cavity and paranasal sinuses [9-13]. Ovarian TCS is thought to originate from germ cells [9-13].

Herein, we report a unique uterine cervical tumor with carcinosarcomatous and teratomatous features, histologically resembling sinonasal TCS.

\section{Case presentation}

\section{Clinical history}

A 45-year-old Japanese woman, gravida 2, para 2, with no remarkable medical history, presented to our hospital for evaluation of a cervical lesion. At her first visit, a gynecologist was not able to detect a mass, but 3 weeks later, the gynecologist found a large polypoid mass on the cervix (Fig. 1a). The patient's laboratory data, including serum tumor markers (carbohydrate antigen 125, alphafetoprotein, human chorionic gonadotropin, carbohydrate antigen 19-9, and carcinoembryonic antigen), were within normal limits. Magnetic resonance imaging revealed a mass on the cervix measuring $28 \times 27 \times 20 \mathrm{~mm}$ (Fig. 1b), with no contrast enhancement. ${ }^{18}$ F-fluorodeoxy-D-glucosepositron emission tomography revealed an abnormal uptake in the polyp and no significant findings on the uterine corpus, bilateral adnexa, and other organs. The polypectomy specimen histologically suggested a malignant germ cell tumor. Subsequently, the patient underwent total abdominal hysterectomy with bilateral salpingo-oophorectomy. Following surgery, she received radiotherapy and has been free of disease for 13 months.

\section{Pathologic findings \\ Macroscopic findings}

The cervical polyp was dark in color and pedunculated, measuring $33 \times 30 \times 25 \mathrm{~mm}$, with a red-tan cut surface due to massive hemorrhage (Fig. 1c). The hysterectomy specimen revealed the cervix almost entirely occupied by a yellow-white solid tumor, measuring $45 \times 40 \times 17 \mathrm{~mm}$ (Fig. 1d).

\section{Microscopic findings}

The cervical tumor showed both epithelial and mesenchymal tissues consisting of immature glandular cells (Fig. 2b), neuroepithelial tissue (Fig. 2c), small blue cells with neuroendocrine differentiation (Fig. 2d), hyaline cartilage (Fig. 3a), and rhabdomyosarcoma (Fig. 3b). Among these tissues, the most prominent component was neuroepithelium, and the tumor contained no immature squamous epithelium, which is common in sinonasal TCS. The tumor had invaded more than half the cervical stroma, but with no involvement of the parametrium or vagina. The tumor had prominent lymphovascular infiltration and

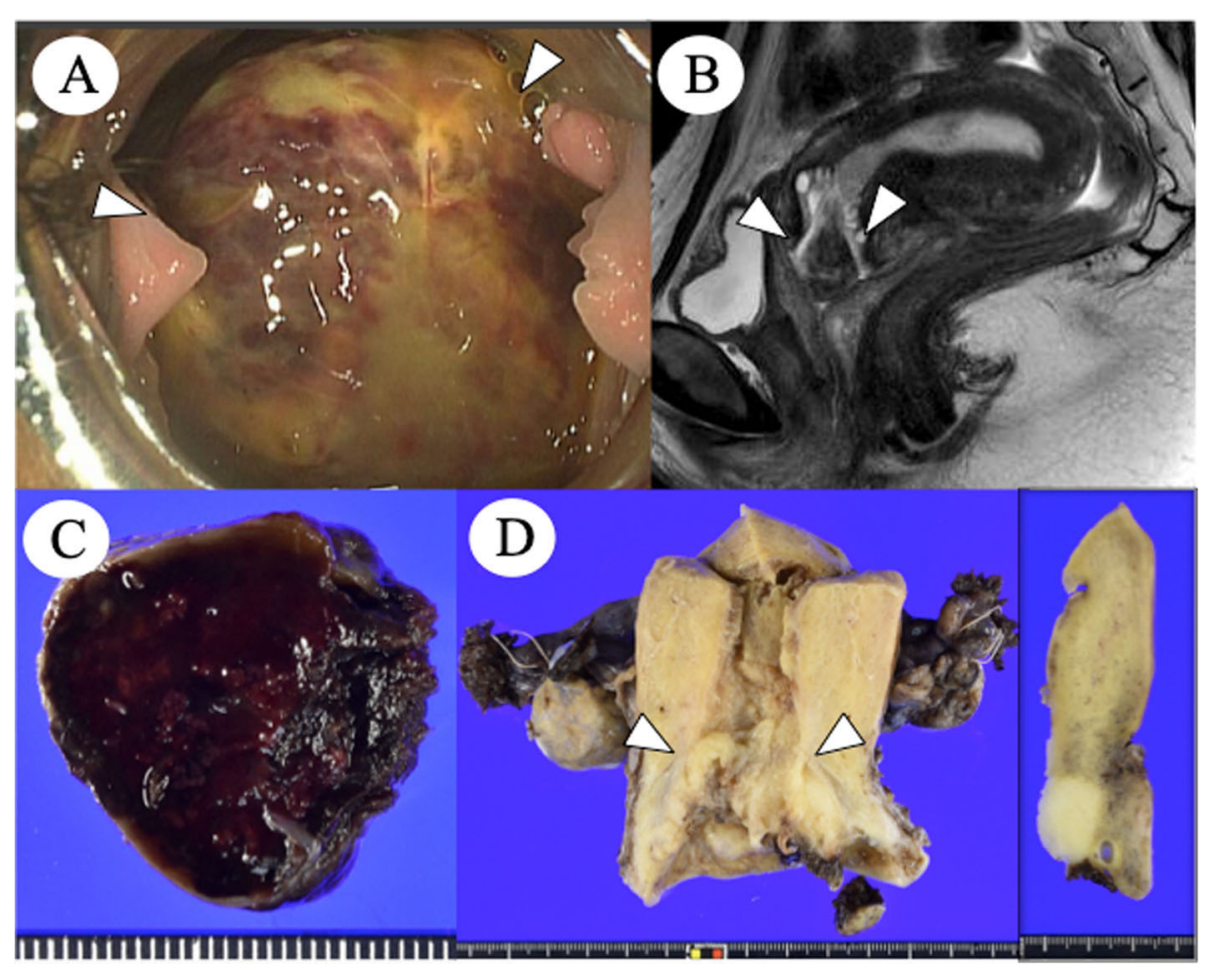

Fig. 1 a Colposcopic image of the cervical mass (arrowhead). b Sagital magnetic resonance image of the low-intensity cervical mass (arrowhead). c Cut section of the cervical mass. d Yellow-white surgical specimen (arrowhead) from the uterus. Inset: cut surface of the uterus 

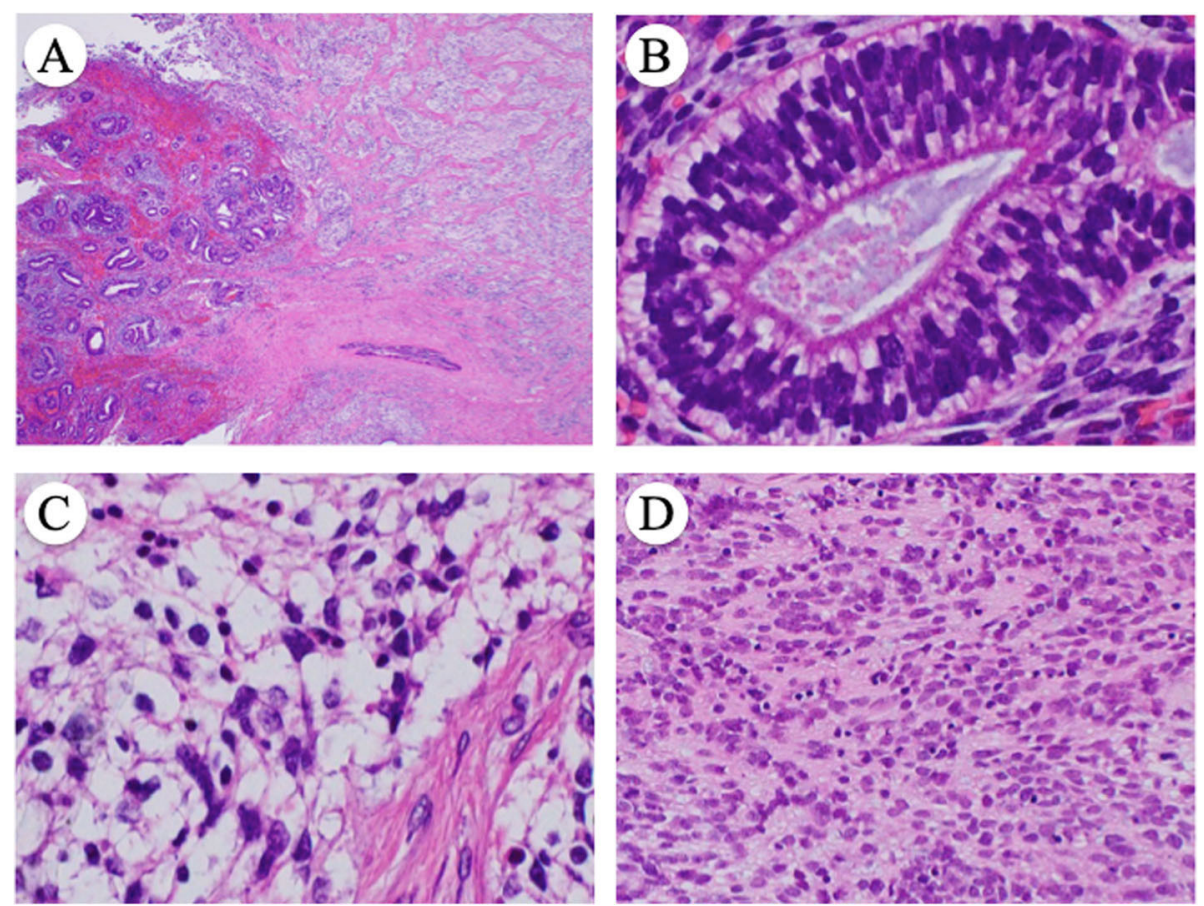

Fig. 2 a Abrupt transition between carcinosarcomatous (left) and neuroectodermal (right) components (magnification $\times 100)$. b Immature epithelial gland (magnification $\times 400)$. c Neuroectodermal tissue (magnification $\times 400)$. $\mathbf{d}$ Small blue cells with neuroendocrine differentiation (magnification $\times 200)$
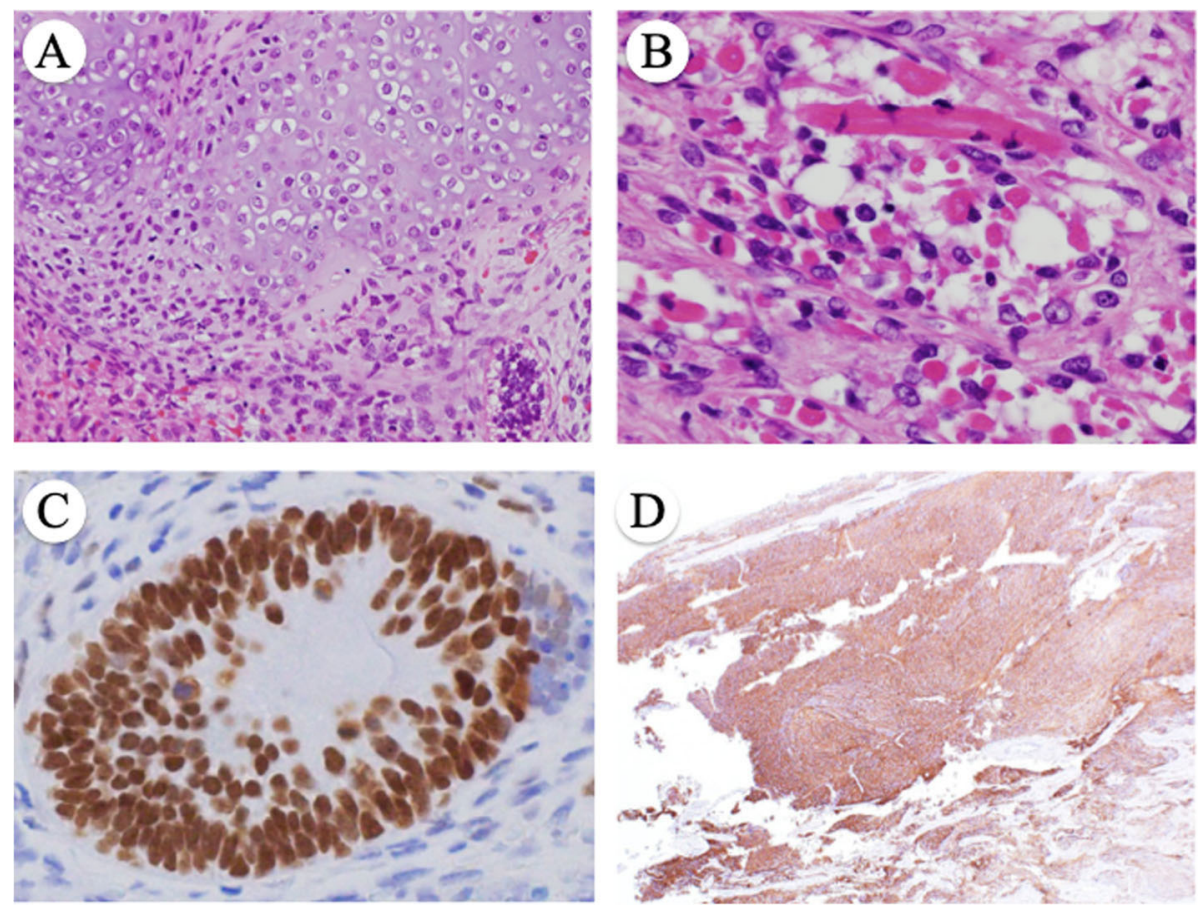

Fig. 3 a Hyaline cartilage (magnification $\times 200$ ). b Striated muscle (magnification $\times 400)$ ). Immunostained images of (c) SALL4 (magnification $\times$ 400) and (d) synaptophysin (magnification $\times 200$ ) 
extended to the endometrium. Bilateral fallopian tubes and ovaries were free of the tumor.

\section{Immunohistochemical features}

Table 1 shows a summary of the immunohistochemical staining. Immature glandular epithelium stained positive for Sal-like protein 4 (SALL4) (Fig. 3c), glypican-3, caudal-type homeobox transcription factor 2 (CDX-2) (focally), and CD99, but negative for octamer-binding transcription factor 4 (Oct-4) and estrogen receptor. The neuroepithelium stained positive for neuron-specific enolase, synaptophysin (Fig. 3d), and CD99, but negative for cytokeratin and Oct-4.

\section{Cytogenetic analysis with FISH}

Human papillomavirus (HPV) infection was not detected by in situ hybridization (GenPoint HPV; Dako), and no evidence of ESWR1 gene rearrangement was detected by fluorescence in situ hybridization (FISH) (VysisLSIESWR1 Dual Color Break Apart Probe). Furthermore, neither isochromosome $12 \mathrm{p}(\mathrm{i}(12 \mathrm{p}))$ nor $12 \mathrm{p}$ amplification was observed by FISH (Cytocell 12pter Subtelomere Specific Probe / Cytocell Chromosome 12 Alfa Satellite Probe) (Fig. 4). Based on these findings, the tumor was diagnosed as TCS of the uterine cervix, FIGO stage IB2 (pT1b2 pNX cM0).

\section{Discussion}

The present case is a unique cervical cancer, consisting of neuroepithelial components positive for CD99 and synaptophysin, immature / atypical intestinal glands positive for SALL4 and CDX-2, cartilage cells, and rhabdomyosarcoma, resembling sinonasal TCS. There have been less than 100 cases of TCS reported in the literature to date, most of which were observed to occur in the nasal cavity or paranasal sinuses [8], but were also in

Table 1 Immunohistochemical profile of each component

\begin{tabular}{|c|c|c|c|c|}
\hline Marker & Neuroendocrine Tissue & Glandular Epithelium & Stromal Tissue & Neuroepithelium \\
\hline SALL4 & $+($ focal $)$ & + & - & $+($ focal $)$ \\
\hline Glypican-3 & + (focal) & + & - & + (focal) \\
\hline Oct-4 & - & - & - & - \\
\hline S-100 & - & - & - & - \\
\hline PLAP & - & - & - & - \\
\hline NSE & + & - & - & + \\
\hline Neurofilament & $+($ focal $)$ & - & - & - \\
\hline GFAP & - & - & - & - \\
\hline CD56 & + & + & - & + \\
\hline CD57 & + & - & + & + \\
\hline CD99 & + & + & + & + \\
\hline Synaptophysin & + & - & - & + \\
\hline Chromogranin A & - & - & - & - \\
\hline AFP & - & - & - & - \\
\hline TTF-1 & - & - & - & - \\
\hline CDX-2 & - & $+($ focal $)$ & - & - \\
\hline WT1 & - & $+($ focal $)$ & - & - \\
\hline ER & - & - & - & - \\
\hline CD10 & - & - & - & - \\
\hline PAX-8 & $+($ focal $)$ & + & + & $+($ focal $)$ \\
\hline$\beta$-catenin & - & +(membrane) & - & - \\
\hline AE1/AE3 & - & + & - & - \\
\hline CD34 & - & - & + & - \\
\hline Desmin & - & - & + in STMC & - \\
\hline a-SMA & - & - & - & - \\
\hline MyoD1 & - & - & + in STMC & - \\
\hline
\end{tabular}

AFP, Alpha-fetoprotein; $a$-SMA, Alpha-smooth muscle actin; CDX-2, Caudal-type homeobox transcription factor 2; ER, Estrogen receptor; GFAP, Glial fibrillary acidic protein; NSE, Neuron-specific enolase; Oct-4, Octamer-binding transcription factor 4; PLAP, Placental alkaline phosphatase; SALL4, Sal-like protein 4; STMC, Striated muscle cells; TTF-1, Thyroid transcription factor 1 ; WT1, Wilms' tumor 1 


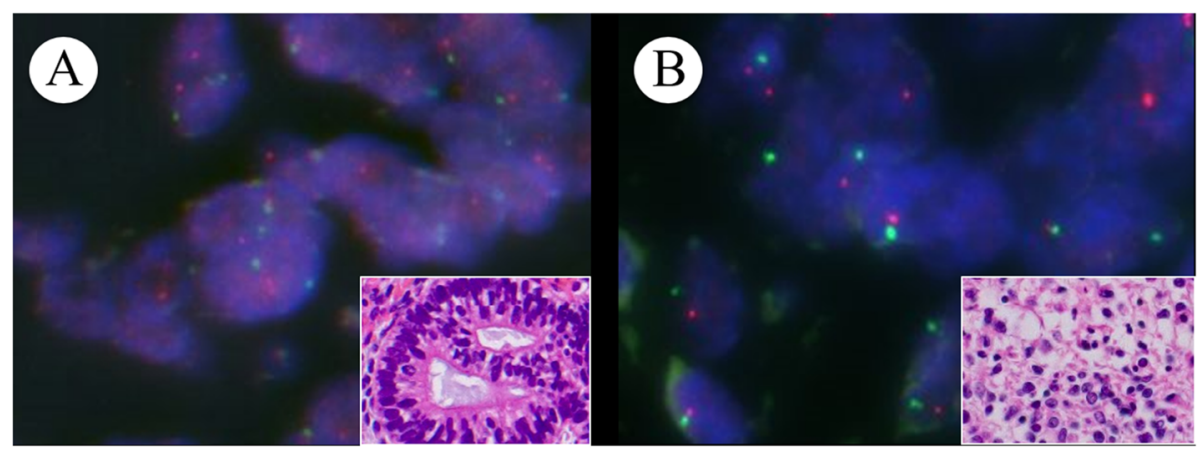

Fig. 4 Chromosome 12p to centromere ratio is not increased by FISH (green signals-12p telomere; red signals-12 centromere): (a) in immature / atypical glands (inset, H\&E stain), and (b) in neuroepithelium (inset, H\&E stain)

female reproductive organs, especially the ovary [9-13] (Table 2). TCS has several differential diagnoses such as carcinosarcoma, immature teratoma, teratoma with malignant transformation, and peripheral primitive neuroectodermal tumor, because of its histological heterogeneity.

Currently, teratomas of the ovary and cervix are thought to have a parthenogenetic origin from the oocyte after completion of the first division [5]. Extragonadal teratoma usually arises in midline structures, such as the sacrococcygeal, mediastinal, and sacral regions, and rarely arises in the uterine cervix [5]. Grayson et al. [2] reported that in 8 cases with uterine cervical carcinosarcoma, HPV was detected in all. However, the present case showed no HPV infection, which was confirmed by the negative reactions of immunohistochemical staining for $\mathrm{p} 16^{\mathrm{INK} 4 \mathrm{a}}$ and HPV in situ hybridization. Immature teratoma is defined by the presence of neuroepithelial tubules and rosettes. In the present case, however, the tumor predominantly consisted of neuroepithelial tissues, but lacked neuroepithelial tubules or rosettes. In addition, the tumor was immunohistochemically negative for Oct-4, which is usually positive for immature teratoma grade $2 / 3$ [14]. In this case, malignant transformation of mature teratoma could be ruled out because of an absence of continuity between malignant or immature components and mature teratomatous tissues. Peripheral primitive neuroectodermal tumor was considered unlikely because ESWR1 rearrangement was not observed.

In the present case, immature / atypical intestinal glands and neuroepithelium were positive for SALL4, which is known as a sensitive and specific marker of germ cell tumors [15]. This suggests that this tumor is germ cell derived. In the pure mature or immature teratoma, there is the presence of $i(12 p)$ and $12 p$ amplification in non-teratoma germ cell components in ovarian or sacrococcygeal tissues [16, 17]. However, this case showed neither $i(12 p)$ nor $12 p$ amplification. The absence of cytogenetic abnormalities in this case suggests that the teratomatous components have an analogous histogenesis compared to the pure ovarian teratoma.

The histogenesis of cervical TCS remains to be clarified, but teratoid carcinosarcoma of the ovary has been reported to show components derived from germ cells

Table 2 Teratocarcinosarcomas of the female genital tract

\begin{tabular}{|c|c|c|c|c|c|c|c|}
\hline $\begin{array}{l}\text { Author } \\
\text { [year] }\end{array}$ & $\begin{array}{l}\text { Age, } \\
y\end{array}$ & Primary & Diagnosis & Treatment & Stage & Histology & Outcome \\
\hline $\begin{array}{l}\text { Ehrmann RL } \\
\text { [1990] }\end{array}$ & 62 & Ovary & TCS & $\begin{array}{l}\text { Surgery }+ \\
\text { chemotherapy }\end{array}$ & III & SCC, adenocarcinoma, chondrosarcoma, rhabdomyosarcoma & $\begin{array}{l}\mathrm{DOD} \\
27 \mathrm{mo}\end{array}$ \\
\hline $\begin{array}{l}\text { Tanimoto A } \\
\text { [2001] }\end{array}$ & 59 & Ovary & TCS & Surgery & IV & SCC, adenocarcinoma, chondrosarcoma & DOD \\
\hline $\begin{array}{l}\text { Garcia-Garvis } \\
\text { OF [2008] }\end{array}$ & 69 & Ovary & TCS & Surgery & IV & $\begin{array}{l}\text { SCC, adenocarcinoma, chondrosarcoma, rhabdomyosarcoma, } \\
\text { osteosarcoma, liposarcoma, yolk-sac tumor }\end{array}$ & DOD \\
\hline $\begin{array}{l}\text { Matsuura Y } \\
{[2010]}\end{array}$ & 40 & Ovary & TCS & $\begin{array}{l}\text { Surgery + } \\
\text { chemotherapy }\end{array}$ & $\|$ & Adenocarcinoma, chondrosarcoma, endometrial stromal sarcoma & $\begin{array}{l}\mathrm{DOD} \\
46 \mathrm{mo}\end{array}$ \\
\hline $\begin{array}{l}\text { Coutney F } \\
{[2019]}\end{array}$ & 55 & Ovary & TCS & $\begin{array}{l}\text { Surgery }+ \\
\text { chemotherapy }\end{array}$ & IC & $\begin{array}{l}\text { Adenocarcinoma, carcinosarcoma, dysgerminoma, primitive retinal } \\
\text { tissue } \\
\text { primitive neuroepithelial tissue, fetal cartilage }\end{array}$ & $\begin{array}{l}\text { DOD } \\
14 \mathrm{mo}\end{array}$ \\
\hline $\begin{array}{l}\text { Present case } \\
\text { [2019] }\end{array}$ & 45 & Cervix & TCS & $\begin{array}{l}\text { Surgery }+ \\
\text { radiation }\end{array}$ & IB2 & $\begin{array}{l}\text { Glial-like tissues, immature intestinal glands, cartilage, } \\
\text { neuroepithelial tissue, rhabdomyosarcoma }\end{array}$ & $\begin{array}{l}\text { NED } \\
13 \mathrm{mo}\end{array}$ \\
\hline
\end{tabular}

TCS, Teratocarcinosarcoma; SCC, Squamous cell carcinoma; DOD, Died of disease; NED, No evidence of disease 
[9-13]. Although germ cells are not usually found in the uterine cervix, extragonadal germ cell tumors rarely occur in the uterine cervix [3-5]. The cases of ovarian TCS showed a poor clinical outcome mainly because they were at the advanced stage. Radical surgical resection followed by radiotherapy is the most common treatment option for sinonasal TCS [7, 8]; therefore, a combination of surgery and radiotherapy may be expected to be effective for the present case with TCS.

\section{Conclusion}

This is the first case report of uterine cervical TCS that may have originated from germ cells, but with a histogenetic pathway different from pure mature teratoma of the ovary.

\section{Abbreviations}

CDX-2: Caudal type homeobox transcription factor 2; FISH: Fluorescence in situ hybridization; HPV: Human papillomavirus; Oct-4: Octamer-binding transcription factor 4; SALL4: Sal-like protein 4; TCS: Teratocarcinosarcoma

\section{Acknowledgments}

We wish to thank the colleagues in the pathology laboratories at Saitama Medical University International Medical Center for their excellent technical assistance; Special thanks to Drs. Takako Kiyokawa and Yoshiki Mikami for their expert pathologic opinions.

\section{Authors' contributions \\ $\mathrm{KI}$ analyzed the data and wrote the manuscript as a major contributor. AO and MM contributed to the management of the patient. MiY carried out the histopathologic evaluation and helped to write the manuscript. EK reviewed the magnetic resonance imaging findings. MaY participated in writing the final manuscript as a corresponding author. All authors have read and approved the final manuscript.}

\section{Funding}

This work was supported by Hidaka Research Projects in Saitama Medical University (grant No. 30-D-1-3) and grants-in-aid from the Ministry of Education, Science, Sports and Culture of Japan (research project No. 18 K06997).

\section{Availability of data and materials}

The datasets used and / or analyzed during the current study are available from the corresponding author upon reasonable request.

\section{Ethics approval and consent to participate}

Not applicable.

\section{Consent for publication}

We obtained written informed consent from the patient for the publication of this case report and accompanying images. A copy of this written consent is available for review by the Editor-in-Chief of this journal.

\section{Competing interests}

The authors declare that they have no competing interests.

\section{Author details}

'Department of Pathology, Saitama Medical University International Medical Center, 1397-1 Yamane, Hidaka-City, Saitama 350-1298, Japan. ${ }^{2}$ Department of Obstetrics and Gynecology, Oita University Faculty of Medicine, Yufu-shi, Oita 879-5593, Japan. ${ }^{3}$ Department of Gynecologic Oncology, Saitama Medical University International Medical Center, 1397-1 Yamane, Hidaka-City, Saitama 350-1298, Japan. ${ }^{4}$ Department of Diagnostic Radiology, Saitama Medical University Hospital, Moroyama, Saitama 350-0495, Japan.
Received: 15 May 2019 Accepted: 12 September 2019

Published online: 04 November 2019

\section{References}

1. Abell MR, Ramirez JA. Sarcomas and carcinosarcomas of the uterine cervix. Cancer. 1973;31:1176-92.

2. Grayson W, Taylor LF, Cooper K. Carcinosarcoma of the uterine cervix: a report of eight cases with immunohistochemical analysis and evaluation of human papillomavirus status. Am J Surg Pathol. 2001;25:338-47.

3. Cortes J, Llompart M, Rossello JJ, Rifa J, Mas J, Anglada P, Serra C, Cartana J. Immature teratoma primary of the uterine cervix. First case report. Eur J Gynaecol Onc. 1990;11:37-42.

4. Panesar NK, Sidhu JS. Uterine cervical teratoma with divergent neuroepithelial differentiation and development of an oligodendroglioma: report of a case and review of the literature. Ann Diagn Pathol. 2007:11:293-6.

5. Oosterhuis JW, Stoop H, Honecker F, Looijenga LH. Why human extragonadal germ cell tumours occur in the midline of the body: old concepts, new perspectives. Int J Androl 2007;30:256-263; discussion 263-54.

6. Shanmugaratnam K, Kunaratnam N, Chia KB, Chiang GS, Sinniah R. Teratoid carcinosarcoma of the paranasal sinuses. Pathology. 1983;15:413-9.

7. Heffner DK, Hyams VJ. Teratocarcinosarcoma (malignant teratoma?) of the nasal cavity and paranasal sinuses. A clinicopathologic study of 20 cases. Cancer. 1984;53:2140-54.

8. Misra P, Husain Q, Svider PF, Sanghvi S, Liu JK, Eloy JA. Management of sinonasal teratocarcinosarcoma: a systematic review. Am J Otolaryngol. 2014:35:5-11.

9. Ehrmann RL, Weidner N, Welch WR, Gleiberman I. Malignant mixed mullerian tumor of the ovary with prominent neuroectodermal differentiation (teratoid carcinosarcoma). Int J Gynecol Pathol. 1990;9:272-82.

10. Tanimoto A, Arima N, Hayashi R, Hamada T, Matsuki Y, Sasaguri Y. Teratoid carcinosarcoma of the ovary with prominent neuroectodermal differentiation. Pathol Int. 2001;51:829-32

11. Garcia-Galvis OF, Cabrera-Ozoria C, Fernandez JA, Stolnicu S, Nogales FF. Malignant Mullerian mixed tumor of the ovary associated with yolk sac tumor, neuroepithelial and trophoblastic differentiation (teratoid carcinosarcoma). Int J Gynecol Pathol. 2008;27:515-20.

12. Matsuura Y, Kitajima M, Hachisuga T, Tanimoto A, Okura N, Kihara I. Malignant mixed mullerian tumor with malignant neuroectodermal components (teratoid carcinosarcoma) of the ovary: report of a case with clinicopathologic findings. J Obstet Gynaecol Res. 2010;36:907-11.

13. Fox C, Allen N, Schimp V, Maksem J. Ovarian Teratoid Carcinosarcoma is an aggressive tumor of probable Mullerian derivation with a Carcinosarcomatous and mixed germ-cell morphology. Case Rep Oncol. 2019:12:241-7.

14. Abiko K, Mandai M, Hamanishi J, Matsumura N, Baba T, Horiuchi A, Mikami Y, Yoshioka S, Wakasa T, Shiozawa T, et al. Oct4 expression in immature teratoma of the ovary: relevance to histologic grade and degree of differentiation. Am J Surg Pathol. 2010:34:1842-8.

15. Cao D, Guo S, Allan RW, Molberg KH, Peng Y. SALL4 is a novel sensitive and specific marker of ovarian primitive germ cell tumors and is particularly useful in distinguishing yolk sac tumor from clear cell carcinoma. Am J Surg Pathol. 2009:33:894-904

16. Poulos C, Cheng L, Zhang S, Gersell DJ, Ulbright TM. Analysis of ovarian teratomas for isochromosome 12p: evidence supporting a dual histogenetic pathway for teratomatous elements. Mod Pathol. 2006;19:766-71.

17. Emerson RE, Kao CS, Eble JN, Grignon DJ, Wang M, Zhang S, Wang X, Fan R, Masterson TA, Roth LM, Cheng L. Evidence of a dual histogenetic pathway of sacrococcygeal teratomas. Histopathology. 2017;70:290-300.

\section{Publisher's Note}

Springer Nature remains neutral with regard to jurisdictional claims in published maps and institutional affiliations. 